\title{
Maximum Principles for Discrete and Semidiscrete Reaction-Diffusion Equation
}

\author{
Petr Stehlík and Jonáš Volek \\ Department of Mathematics and NTIS, Faculty of Applied Sciences, University of West Bohemia, Univerzitni 8, \\ 30614 Pilsen, Czech Republic
}

Correspondence should be addressed to Petr Stehlík; pstehlik@kma.zcu.cz

Received 24 March 2015; Accepted 29 July 2015

Academic Editor: Cengiz Çinar

Copyright (C) 2015 P. Stehlík and J. Volek. This is an open access article distributed under the Creative Commons Attribution License, which permits unrestricted use, distribution, and reproduction in any medium, provided the original work is properly cited.

We study reaction-diffusion equations with a general reaction function $f$ on one-dimensional lattices with continuous or discrete time $u_{x}^{\prime}\left(\right.$ or $\left.\Delta_{t} u_{x}\right)=k\left(u_{x-1}-2 u_{x}+u_{x+1}\right)+f\left(u_{x}\right), x \in \mathbb{Z}$. We prove weak and strong maximum and minimum principles for corresponding initial-boundary value problems. Whereas the maximum principles in the semidiscrete case (continuous time) exhibit similar features to those of fully continuous reaction-diffusion model, in the discrete case the weak maximum principle holds for a smaller class of functions and the strong maximum principle is valid in a weaker sense. We describe in detail how the validity of maximum principles depends on the nonlinearity and the time step. We illustrate our results on the Nagumo equation with the bistable nonlinearity.

\section{Introduction}

Reaction-diffusion equation $\partial_{t} u=k \partial_{x x} u+f(u)$ (sometimes called FKPP equation, which abbreviates Fisher, Kolmogorov, Petrovsky, Piskounov) serves as a nonlinear model to describe a class of (biological, chemical, economic, and so forth) phenomena in which two factors are combined. Firstly, the diffusion process causes the concentration of a substance (animals, wealth, and so forth) to spread in space. Secondly, a local reaction leads to dynamics based on the concentration values.

For the sake of applications and correctness of numerical procedures it makes sense to consider partially or fully discretized reaction-diffusion equation. In certain situations (e.g., spatially structured environment) it is natural to study reaction-diffusion equations with discretized space variable and continuous time (we refer to it as a semidiscrete problem and use $\left.u_{x}(t)=u(x, t)\right)$ :

$$
\begin{array}{r}
u_{x}^{\prime}(t)=k\left(u_{x-1}(t)-2 u_{x}(t)+u_{x+1}(t)\right)+f\left(u_{x}(t)\right), \\
x \in \mathbb{Z}, t \in[0,+\infty),
\end{array}
$$

or, for example, if nonoverlapping populations are considered, with both time and space variables being discrete (a discrete problem, $\left.u_{x, t}:=u(x, t)\right)$ :

$$
\begin{array}{r}
\frac{u_{x, t+h}-u_{x, t}}{h}=k\left(u_{x-1, t}-2 u_{x, t}+u_{x+1, t}\right)+f\left(u_{x, t}\right), \\
x \in \mathbb{Z}, t \in\{0, h, 2 h, \ldots\} .
\end{array}
$$

Examples of such phenomena are chemical reactions related to crystal formation, see Cahn [1], or myelinated nerve axons, see Bell and Cosner [2] and Keener [3]. Existence and nonexistence of travelling waves in those models have been recently studied in Chow [4], Chow et al. [5], and Zinner [6] mostly with the cubic (or bistable, double-well) nonlinearities of the form $f(u)=\lambda u(u-a)(1-u)$, with $\lambda>0$ and $a \in(0,1)$ (this special case of FKPP equation is being referred to as Nagumo equation). In contrast, various reaction functions have been proposed in models without spatial interaction, for example, Xu et al. [7].

Motivated by these facts, we allow for a general form of the reaction function $f$ in this paper (i.e., we do not restrict ourselves to cubic nonlinearities). We prove a priori estimates 
for discrete reaction-diffusion equation (2) and then use Euler method to show their validity for semidiscrete reactiondiffusion equation (1). Whereas the maximum principles in the semidiscrete case exhibit similar features to those of continuous reaction-diffusion model (i.e., they hold under similar assumptions), in the discrete case the weak maximum principle holds for a smaller class of functions and the strong maximum principle is only valid in a weaker sense involving the domain of dependence. Finally, we use the maximum principles to get the global existence of solutions of the initial-boundary problem for the semidiscrete case (1). All our results are illustrated in detail in Nagumo equations with a symmetric bistable nonlinearity; that is, we consider problems (1)-(2) with $f(u)=\lambda u\left(1-u^{2}\right)$.

Our motivation is twofold. First, maximum principles could be used to obtain comparison principles (Protter and Weinberger [8]), which in turn could serve as a valuable tool in the study of traveling waves, for example, Bell and Cosner [2]. Moreover, similarly as in the case of (non)existence of traveling wave solutions for Nagumo equations, it has been shown that discrete and semidiscrete structures influence the validity of maximum principles in a significant way. Even the simplest one-dimensional linear problems require additional assumptions on the step size; see Mawhin et al. [9] and Stehlík and Thompson [10]. In the case of partial difference and semidiscrete equations, the strong influence of the underlying structure on maximum principles has been described in the linear case for transport equation in Stehlík and Volek [11] and for diffusion-type equations in Slavík and Stehlík [12] and Friesl et al. [13] (interestingly, the proofs of maximum principles in this case are based on product integration; see Slavík [14]). Finally, simple maximum principles for nonlinear transport equations on semidiscrete domains have been presented in Volek [15].

In the classical case, maximum principles for diffusion (and parabolic) equations go back to Picone [16] and Levi [17]. Strong maximum principles were later established by Nirenberg [18] and a survey of various versions and applications could be found in a classical monograph Protter and Weinberger [8].

This paper is segmented in the following way. In Section 2, we briefly summarize results for the classical reactiondiffusion equation. Next, we prove weak and strong maximum principles for the discrete case (2) (Sections 3 and 4). In the case of the initial-boundary value problem for the semidiscrete equation (1) we provide local existence results (Section 5) and maximum principles (Section 6) which we consequently apply to get global existence of solutions in Section 7. Our results are then applied to the Nagumo equation with a symmetric bistable nonlinearity, that is, problems (1)-(2) with $f(u)=\lambda u\left(1-u^{2}\right)$, in Section 8 .

\section{Reaction-Diffusion Partial Differential Equation}

In order to motivate and compare our results for the reactiondiffusion equations on discrete-space domains with the classical reaction-diffusion equation we briefly summarize few basic results for the following initial-boundary problem:

$$
\begin{aligned}
\partial_{t} u(x, t) & =k \partial_{x x} u(x, t)+f(x, t, u(x, t)), \\
x \in(a, b), t \in \mathbb{R}^{+}, k>0, & x \in[a, b], \\
u(x, 0) & =\varphi(x), \quad x \in \mathbb{R}_{0}^{+}, \\
u(a, t)=\xi_{a}(t), \quad t \in \mathbb{R}^{+}, & t \in \mathbb{R}_{0}^{+},
\end{aligned}
$$

where $f:(a, b) \times \mathbb{R}^{+} \times \mathbb{R} \rightarrow \mathbb{R}$ is a reaction function and $\varphi:[a, b] \rightarrow \mathbb{R}, \xi_{a}, \xi_{b}: \mathbb{R}_{0}^{+} \rightarrow \mathbb{R}$ are initial-boundary conditions satisfying $\varphi(a)=\xi_{a}(0)$ and $\varphi(b)=\xi_{b}(0)$.

The following existence and uniqueness result for (3) can be found, for example, in [19, page 298].

Theorem 1. Let $T>0$ be arbitrary and let $f$ be uniformly Hölder continuous in $x$ and $t$ and Lipschitz in $u$ for $(x, t) \epsilon$ $(a, b) \times(0, T]$. Then for all Hölder continuous initial-boundary conditions $\varphi, \xi_{a}, \xi_{b}$ problem (3) has a unique bounded solution which is defined on $[a, b] \times[0, T]$.

We define the following two numbers for the brevity:

$$
\begin{aligned}
M_{T} & :=\max _{x \in[a, b], t \in[0, T]}\left\{\varphi(x), \xi_{a}(t), \xi_{b}(t)\right\}, \\
m_{T} & :=\min _{x \in[a, b], t \in[0, T]}\left\{\varphi(x), \xi_{a}(t), \xi_{b}(t)\right\} .
\end{aligned}
$$

For the linear diffusion equation (i.e., (3) with $f(x, t, u) \equiv$ 0 ) the maximum principle is proved, for example, in [8, Chapter 3.1]. For the nonlinear problem (3) (i.e., $f(x, t, u) \neq$ 0 ) the following weak maximum principle holds (see [19, Theorem 1]).

Theorem 2. Let $T>0$ be arbitrary and let $f$ be uniformly Hölder continuous in $x$ and $t$ and Lipschitz in $u$ for $(x, t) \epsilon$ $(a, b) \times(0, T]$ and assume that

$$
\begin{aligned}
& f\left(x, t, M_{T}\right) \leq 0 \leq f\left(x, t, m_{T}\right) \\
& \forall(x, t) \in(a, b) \times(0, T] .
\end{aligned}
$$

Let $u$ be a continuous solution of (3) with Hölder continuous initial-boundary conditions $\varphi, \xi_{a}, \xi_{b}$. Then

$$
m_{T} \leq u(x, t) \leq M_{T}
$$

holds for all $(x, t) \in[a, b] \times[0, T]$.

Moreover, the strong maximum principle also holds (see [19, Theorem 2]).

Theorem 3. Let the assumptions of Theorem 2 be satisfied and let $u$ be a solution of (3) on $[a, b] \times[0, T]$. If $u\left(x_{0}, t_{0}\right)=M_{T}$ (or $\left.u\left(x_{0}, t_{0}\right)=m_{T}\right)$ for some $\left(x_{0}, t_{0}\right) \in(a, b) \times(0, T]$ then

$$
\begin{aligned}
u(x, t)= & M_{T} \\
(\operatorname{or} u(x, t) & \left.=m_{T}\right) \\
& \forall(x, t) \in[a, b] \times\left[0, t_{0}\right] .
\end{aligned}
$$




\section{Discrete Reaction-Diffusion Equation: Weak Maximum Principles}

Let us consider the initial-boundary value problem for the discrete reaction-diffusion equation (which could be obtained, e.g., by Euler discretization of (3)):

$$
\begin{aligned}
\frac{u(x, t+h)-u(x, t)}{h \quad}= & k \Delta_{x x}^{2} u(x-1, t) \\
& +f(x, t, u(x, t)), \\
x \in & (a, b)_{\mathbb{Z}}, t \in h \mathbb{N}_{0}, h>0, k>0, \\
u(x, 0) & =\varphi(x), \quad x \in(a, b)_{\mathbb{Z}}, \\
u(a, t) & =\xi_{a}(t), \quad t \in h \mathbb{N}_{0}, \\
u(b, t) & =\xi_{b}(t), \quad t \in h \mathbb{N}_{0},
\end{aligned}
$$

where $f:(a, b)_{\mathbb{Z}} \times h \mathbb{N}_{0} \times \mathbb{R} \rightarrow \mathbb{R}$ is a reaction function, $\varphi:(a, b)_{\mathbb{Z}} \rightarrow \mathbb{R}, \xi_{a}, \xi_{b}: h \mathbb{N}_{0} \rightarrow \mathbb{R}$ are initial-boundary conditions, $h \mathbb{N}_{0}:=\left\{h n, n \in \mathbb{N}_{0}\right\},(a, b)_{\mathbb{Z}}:=(a, b) \cap \mathbb{Z}$, and $\Delta_{x x}^{2} u(x-1, t):=u(x-1, t)-2 u(x, t)+u(x+1, t)$ (for brevity, we assume the space discretization step $h_{x}=1$, but all our results are easily extendable to an arbitrary step $h_{x}>0$ if we use the diffusion constant $\widetilde{k}=k / h_{x}^{2}$ instead of $k$; we discuss this in detail in a specific example at the end of Section 8).

Straightforwardly, problem (8) has a unique solution which is defined in $[a, b]_{\mathbb{Z}} \times h \mathbb{N}_{0}$, since $u(x, t+h)$ is uniquely given by

$$
\begin{aligned}
& u(x, t+h) \\
& = \begin{cases}u(x, t)+h\left(k \Delta_{x x}^{2} u(x-1, t)+f(x, t, u(x, t))\right), & x \in(a, b)_{\mathbb{Z}}, \\
\xi_{a}(t+h), & x=a, \\
\xi_{b}(t+h), & x=b .\end{cases}
\end{aligned}
$$

For $T \in h \mathbb{N}_{0}$, we define the following two numbers:

$$
\begin{aligned}
& M_{T}:=\max _{x \in(a, b)_{\mathbb{Z}}, t \in[0, T]_{h \mathbb{N}_{0}}}\left\{\varphi(x), \xi_{a}(t), \xi_{b}(t)\right\},
\end{aligned}
$$

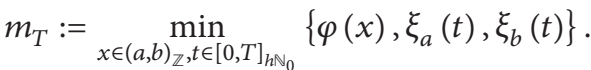

For brevity of the following assertions we formulate the assumption in the reaction function $f$ :

(D) Let $T \in h \mathbb{N}_{0}$ and let $f$ satisfy

$$
\begin{aligned}
& \frac{2 h k-1}{h}\left(u-m_{T}\right) \leq f(x, t, u) \leq \frac{2 h k-1}{h}\left(u-M_{T}\right), \\
& \quad \text { for all } x \in(a, b)_{\mathbb{Z}}, t \in[0, T]_{h \mathbb{N}_{0}} \text { and } u \in\left[m_{T}, M_{T}\right] .
\end{aligned}
$$

Remark 4. The inequalities (12) imply that for all fixed $x$ and $t$ the graph of function $f(x, t, \cdot)$ does not intersect the forbidden area depicted in Figure 1.

Remark 5. Let us notice that for $h \rightarrow 0+$ the slope (2hk1) $/ h$ goes to $-\infty$; that is, the forbidden area from Remark 4 is smaller in the sense of inclusion and it is easier to satisfy assumption $(D)$ if we decrease the time discretization step $h$. We illustrate this fact in Figure 1.

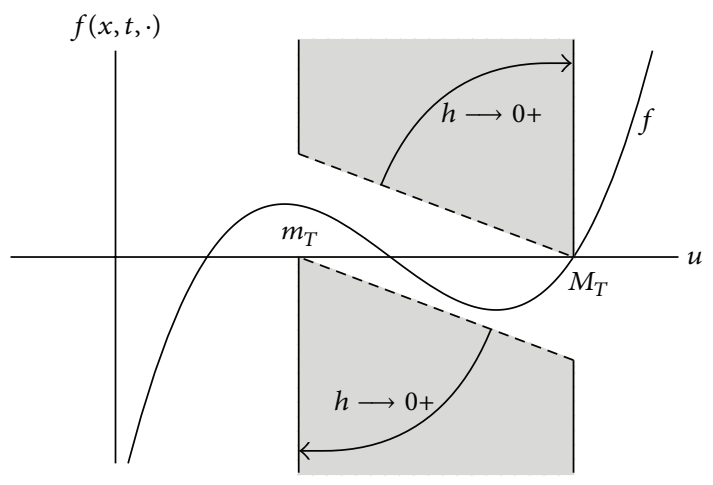

FIGURE 1: The forbidden area for the function $f(x, t, \cdot)$ in assumption $(D)$. The change of this area if $h \rightarrow 0+$. The slope of the dashed line is given by $2 k-1 / h$; see assumption $(D)$.

Proposition 6. Assume that $m_{T}<M_{T}$. If $h>1 / 2 k$ then $(D)$ does not hold for any function $f$.

Note that the inequality $h \leq 1 / 2 k$ is the necessary condition for the validity of maximum principles even in the linear case; see, for example, [13, Theorem 2.4].

Proof. If $h>1 / 2 k$ (i.e., $2 h k-1>0$ ) then from (12) there should be

$$
\begin{aligned}
0 & <\frac{2 h k-1}{h}\left(u-m_{T}\right) \leq f(x, t, u) \\
& \leq \frac{2 h k-1}{h}\left(u-M_{T}\right)<0 \text { for } u \in\left(m_{T}, M_{T}\right),
\end{aligned}
$$

a contradiction.

Remark 7. Notice that if $m_{T}=M_{T}$ then $(D)$ implies that $f\left(x, t, m_{T}\right)=f\left(x, t, M_{T}\right)=0$ for all $x \in(a, b)_{\mathbb{Z}}$ and $t \in[0, T]_{h \mathbb{N}_{0}}$. This situation corresponds to the case of the constant initial-boundary conditions $\varphi(x) \equiv M_{T}$ and $\xi_{a}(t) \equiv$ $\xi_{b}(t) \equiv M_{T}$. From $f\left(x, t, M_{T}\right)=0$ and from (9) there is

$$
u(x, t)=\varphi(x)=M_{T} \text { for } t \in[0, T]_{h \mathbb{N}_{0}} .
$$

Now we state an auxiliary lemma which is crucial in the proof of the maximum principle.

Lemma 8. Let $T \in h \mathbb{N}_{0}$, let function $f$ satisfy $(D)$, and let $u$ be the unique solution of (8). Then for all $x \in[a, b]_{\mathbb{Z}}$ and for all $t \in[0, T)_{h \mathbb{N}_{0}}$

$$
\begin{aligned}
m_{T} \leq u(x, t) \leq & M_{T} \\
& \text { implies that } m_{T} \leq u(x, t+h) \leq M_{T} .
\end{aligned}
$$

Proof. For the sake of brevity, we only show that $u(x, t+h) \leq$ $M_{T}$. The inequality $m_{T} \leq u(x, t+h)$ can be proved in the same way.

Let $t \in h \mathbb{N}_{0}, t<T$, be arbitrary. Then $u(a, t+h)=\xi_{a}(t+$ $h) \leq M_{T}$ and $u(b, t+h)=\xi_{b}(t+h) \leq M_{T}$ trivially from 
the definition of $M_{T}$ (10) (recall that $t<T$, i.e., $t+h \leq T$ ). If $x \in(a, b)_{\mathbb{Z}}$ then we can estimate

$$
\begin{aligned}
& u(x, t+h)=u(x, t)+h(k u(x-1, t)-2 k u(x, t) \\
& \quad+k u(x+1, t)+f(x, t, u(x, t))) \leq 2 h k M_{T}+(1 \\
& \quad-2 h k) u(x, t)+h f(x, t, u(x, t)) .
\end{aligned}
$$

Thanks to the assumptions (12) and $m_{T} \leq u(x, t) \leq M_{T}$ we get

$$
h f(x, t, u(x, t)) \leq(2 h k-1)\left(u(x, t)-M_{T}\right) .
$$

Therefore,

$$
\begin{aligned}
u(x, t+h) \leq & 2 h k M_{T}+(1-2 h k) u(x, t) \\
& +h f(x, t, u(x, t)) \\
\leq & 2 h k M_{T}+(1-2 h k) u(x, t) \\
& +(2 h k-1)\left(u(x, t)-M_{T}\right)=M_{T} .
\end{aligned}
$$

The weak maximum principle follows immediately.

Theorem 9. Let $T \in h \mathbb{N}_{0}$ be arbitrary, let function $f$ satisfy (D), and let $u$ be the unique solution of (8). Then

$$
m_{T} \leq u(x, t) \leq M_{T}
$$

holds for all $x \in[a, b]_{\mathbb{Z}}$ and $t \in[0, T]_{h \mathbb{N}_{0}}$.

Proof. From (10) to (11) we get $m_{T} \leq u(x, 0) \leq M_{T}$ for all $x \in[a, b]_{\mathbb{Z}}$. Immediately, Lemma 8 yields that (19) holds for all $x \in[a, b]_{\mathbb{Z}}$ and $t \in[0, T]_{h \mathbb{N}_{0}}$.

Remark 10. If the reaction function $f$ does not satisfy the inequalities (12) we can find a counterexample that the maximum principle does not hold in general. For example, let us consider (8) with $a=-1, b=1, t \in \mathbb{N}_{0}$, and $\varphi(x) \equiv 0$, $\xi_{a}(t) \equiv \xi_{b}(t) \equiv 0$. Let us assume that, for example, the latter inequality in (12) does not hold; that is,

$$
f(0, t, 0)>\frac{2 h k-1}{h}\left(0-M_{T}\right)=0,
$$

for some $t \in \mathbb{N}_{0}$. Assuming without loss of generality that $t=$ 0 , then the maximum principle is straightforwardly violated since

$$
\begin{aligned}
u(0,1)= & k u(-1,0)+(1-2 k) u(0,0)+k u(1,0) \\
& +f(0,0,0)=f(0,0,0)>0=M_{T} .
\end{aligned}
$$

In certain cases, the function $f$ could fail to satisfy $(D)$ but could still provide a priori bounds for solutions of (8) if the following inequalities hold.

$\left(D^{\prime}\right)$ Let $T \in h \mathbb{N}_{0}$ and let there exist $S \geq M_{T}$ and $R \leq m_{T}$ such that

$$
\frac{2 h k-1}{h}(u-R) \leq f(x, t, u) \leq \frac{2 h k-1}{h}(u-s) \text {, }
$$

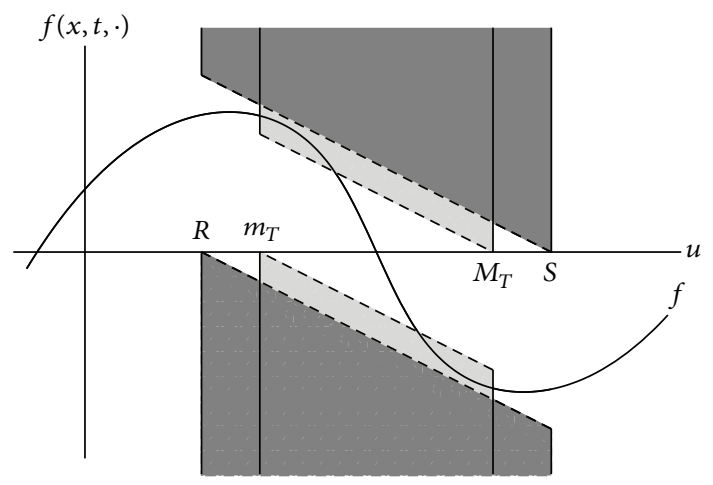

FIgURE 2: The example of the function $f$ that does not satisfy $(D)$ but satisfies $\left(D^{\prime}\right)$ for some constants $R, S$. Such a function consequently provides a priori bounds for solutions of (8) in the sense of Theorem 11.

for all $x \in(a, b)_{\mathbb{Z}}$ and $t \in h \mathbb{N}_{0}$ such that $0 \leq t \leq T$ and $u \in[R, S]$.

In that case, we obtain a general version of the weak maximum principle (for the illustration of $\left(D^{\prime}\right)$ see Figure 2).

Theorem 11. Let $T \in h \mathbb{N}_{0}$ be arbitrary, let function $f$ satisfy $\left(D^{\prime}\right)$, and let $u$ be the unique solution of (8). Then

$$
R \leq u(x, t) \leq S
$$

holds for all $x \in(a, b)_{\mathbb{Z}}$ and $t \in h \mathbb{N}_{0}$ such that $0 \leq t \leq T$.

Proof. For $t=0$ we have

$$
R \leq m_{T} \leq u(x, 0) \leq M_{T} \leq S, \quad \forall x \in(a, b)_{\mathbb{Z}} .
$$

Now we can proceed analogously as in the proofs of Lemma 8 and Theorem 9 where we use $\left(D^{\prime}\right)$ instead of $(D)$. We omit the details.

Example 12. The set of nonlinear reaction functions $f$ that could be considered in Theorem 9 or 11 includes, for example, (for the detailed analysis with $f(x, t, u)=\lambda u\left(1-u^{2}\right)$ see Section 8)

(i) $f(x, t, u)=-|u|^{p-1} u$ with $p>1$,

(ii) the logistic function $f(x, t, u)=u(1-u)$,

(iii) the bistable nonlinearity $f(x, t, u)=\lambda u(u-a)(1-u)$, $a \in(0,1)$,

(iv) $f(x, t, u)=\lambda u\left(1-u^{p}\right)$ where $p \in \mathbb{N}$,

(v) $f(x, t, u)=-|x| \arctan \left(t^{2} u\right)$.

We state the following two claims that are direct corollaries of Theorem 9 .

Corollary 13. Assume that $\xi_{a}, \xi_{b}$ are bounded. Let $f$ satisfy (D) for all $T>0$. Then the unique solution $u$ of (8) is bounded.

Corollary 14. Assume that $\varphi, \xi_{a}, \xi_{b}$ are nonnegative. Let $f$ satisfy (D) for all $T>0$. Then the unique solution $u$ of (8) is nonnegative. 


\section{Discrete Reaction-Diffusion Equation: Strong Maximum Principle}

As in the case of classical reaction-diffusion equation (3) (Theorem 3) we naturally turn our attention to strong maximum principles. Straightforwardly, the strong maximum principle does not hold in the discrete case in the sense of Theorem 3.

Example 15. Let us consider problem (8) with $x \in[-2,2]_{\mathbb{Z}}$, $t \in \mathbb{N}_{0}, f(x, t, u) \equiv 0$, and $k=1 / 2$ (note that $h=1 / 2 k$ ) and let

$$
\begin{aligned}
\varphi(x) & \equiv M>0, \quad x \in\{-1,0,1\} \\
\xi_{-2}(t) & \equiv \xi_{2}(t) \equiv 0, \quad t \in \mathbb{N}_{0} .
\end{aligned}
$$

Then from (9) we get

$$
\begin{aligned}
u(0,1)= & u(0,0)+k u(-1,0)-2 k u(0,0)+k u(1,0) \\
& +f(0,0,0)=M
\end{aligned}
$$

Analogously, we can deduce that

$$
\begin{aligned}
& u(-2,1)=u(2,1)=0, \\
& u(-1,1)=u(1,1)=\frac{M}{2} .
\end{aligned}
$$

Consequently, the strong maximum principle does not hold.

Nonetheless, given the fact that the values of $u(x, t)$ are given by (9), we can easily construct the domain of dependence of $\left(x_{0}, t_{0}\right)$ :

$$
\begin{aligned}
& \mathscr{D}\left(x_{0}, t_{0}\right):=\left\{(x, t) \in[a, b]_{\mathbb{Z}} \times h \mathbb{N}_{0}: t \leq t_{0}, x=x_{0}\right. \\
& \left.\quad \pm j, \quad j=0,1, \ldots, \frac{t_{0}-t}{h}\right\}
\end{aligned}
$$

and the domain of influence of $\left(x_{0}, t_{0}\right)$ :

$$
\begin{aligned}
& \mathscr{I}\left(x_{0}, t_{0}\right):=\left\{(x, t) \in[a, b]_{\mathbb{Z}} \times h \mathbb{N}_{0}: t \geq t_{0}, x=x_{0}\right. \\
& \left.\quad \pm j, \quad j=0,1, \ldots, \frac{t-t_{0}}{h}\right\} .
\end{aligned}
$$

Considering the following:

$\left(D^{\prime \prime}\right)$ Let $T \in h \mathbb{N}_{0}$ and let $f$ satisfy for all $x \in(a, b)_{\mathbb{Z}}, t \in$ $[0, T]_{h \mathbb{N}_{0}}$ :

$$
\begin{aligned}
& \text { (a) } f(x, t, u)<((2 h k-1) / h)\left(u-M_{T}\right) \text { when } u \in \\
& {\left[m_{T}, M_{T}\right),} \\
& \text { (b) } f(x, t, u)>((2 h k-1) / h)\left(u-m_{T}\right) \text { when } u \in \\
& \left(m_{T}, M_{T}\right], \\
& \text { (c) } f\left(x, t, M_{T}\right) \leq 0 \text { and } f\left(x, t, m_{T}\right) \geq 0,
\end{aligned}
$$

the weaker version of the strong maximum principle follows immediately.
Theorem 16. Assume that the function $f$ satisfies $\left(D^{\prime \prime}\right)$ for all $T \in h \mathbb{N}_{0}$. Let $u$ be the unique solution of $(8)$ and $\left(x_{0}, t_{0}\right) \in$ $[a, b]_{\mathbb{Z}} \times h \mathbb{N}_{0}$.

(1) If $u\left(x_{0}, t_{0}\right)=M_{T}\left(\operatorname{or} u\left(x_{0}, t_{0}\right)=m_{T}\right)$, then $u(x, t)=$ $M_{T}\left(\operatorname{or} u(x, t)=m_{T}\right)$ on $\mathscr{D}\left(x_{0}, t_{0}\right)$.

(2) If $u\left(x_{0}, t_{0}\right)<M_{T}\left(\right.$ or $\left.u\left(x_{0}, t_{0}\right)>m_{T}\right)$, then $u(x, t)<$ $M_{T}\left(\operatorname{or} u(x, t)>m_{T}\right)$ on $\mathscr{I}\left(x_{0}, t_{0}\right)$.

Proof. Let us only focus on the former statement of the theorem; the latter could be proved in very similar way. We show that if the function $f$ satisfies $\left(D^{\prime \prime}\right)$ and $u\left(x_{0}, t_{0}\right)=M_{T}$ for some $x_{0} \in(a, b)_{\mathbb{Z}}, t_{0} \in h \mathbb{N}_{0}, 0<t_{0} \leq T$, then $u\left(x_{0}-1, t_{0}-h\right)=u\left(x_{0}, t_{0}-h\right)=u\left(x_{0}+1, t_{0}-h\right)=M_{T}$. The rest follows by induction.

Assume by contradiction first that $u\left(x_{0}-1, t_{0}-h\right)<M_{T}$ (the case $u\left(x_{0}+1, t_{0}-h\right)<M_{T}$ follows easily). Using this assumption, (9), and Theorem 9 we can estimate

$$
\begin{aligned}
u\left(x_{0}, t_{0}\right)= & h k\left(u\left(x_{0}-1, t_{0}-h\right)+u\left(x_{0}+1, t_{0}-h\right)\right) \\
& +(1-2 h k) u\left(x_{0}, t_{0}-h\right) \\
& +h f\left(x_{0}, t_{0}-h, u\left(x_{0}, t_{0}-h\right)\right) \\
< & 2 h k M_{T}+(1-2 h k) u\left(x_{0}, t_{0}-h\right) \\
& +h f\left(x_{0}, t_{0}-h, u\left(x_{0}, t_{0}-h\right)\right)+M_{T} \\
& -M_{T} \\
= & (1-2 h k)\left(u\left(x_{0}, t_{0}-h\right)-M_{T}\right) \\
& +h f\left(x_{0}, t_{0}-h, u\left(x_{0}, t_{0}-h\right)\right)+M_{T} .
\end{aligned}
$$

Thus, $\left(D^{\prime \prime}\right)$ yields

$$
\begin{aligned}
& (1-2 h k)\left(u\left(x_{0}, t_{0}-h\right)-M_{T}\right) \\
& \quad+h f\left(x_{0}, t_{0}-h, u\left(x_{0}, t_{0}-h\right)\right) \leq 0 .
\end{aligned}
$$

Consequently, there has to be

$$
u\left(x_{0}, t_{0}\right)<M_{T}
$$

a contradiction.

If $u\left(x_{0}, t_{0}-h\right)<M_{T}$, then by the similar procedure as above we obtain

$$
\begin{aligned}
u\left(x_{0}, t_{0}\right) \leq & (1-2 h k)\left(u\left(x_{0}, t_{0}-h\right)-M_{T}\right) \\
& +h f\left(x_{0}, t_{0}-h, u\left(x_{0}, t_{0}-h\right)\right)+M_{T} .
\end{aligned}
$$

Since $u\left(x_{0}, t_{0}-h\right) \in\left[m_{T}, M_{T}\right)$ in this case, $\left(D^{\prime \prime}\right)$ implies that

$$
\begin{aligned}
& (1-2 h k)\left(u\left(x_{0}, t_{0}-h\right)-M_{T}\right) \\
& +h f\left(x_{0}, t_{0}-h, u\left(x_{0}, t_{0}-h\right)\right)<0 .
\end{aligned}
$$

Hence,

$$
u\left(x_{0}, t_{0}\right)<M_{T}
$$

a contradiction.

In the case of nonconstant time discretization $h=h(t)$ we can follow similar techniques and consider $(D)$ (eventually, $\left(D^{\prime}\right)$ or $\left.\left(D^{\prime \prime}\right)\right)$ with $h_{\max }\left(\right.$ or $h_{\text {sup }}$ for $T \rightarrow \infty$ ); see Remark 5 . 


\section{Semidiscrete Reaction-Diffusion Equation: Local Existence}

In this section we study the local existence of the following initial-boundary value problem on semidiscrete domains:

$$
\begin{aligned}
& u_{t}(x, t)=k \Delta_{x x}^{2} u(x-1, t)+f(x, t, u(x, t)), \\
& \qquad x \in(a, b)_{\mathbb{Z}}, t \in \mathbb{R}_{0}^{+}, k>0, \\
& u(x, 0)=\varphi(x), \quad x \in(a, b)_{\mathbb{Z}}, \\
& u(a, t)=\xi_{a}(t), \quad t \in \mathbb{R}_{0}^{+}, \\
& u(b, t)=\xi_{b}(t), \quad t \in \mathbb{R}_{0}^{+},
\end{aligned}
$$

where $u_{t}=\partial_{t} u$ denotes the time derivative, $f:(a, b)_{\mathbb{Z}} \times \mathbb{R}_{0}^{+} \times$ $\mathbb{R} \rightarrow \mathbb{R}$ is a reaction function, $\varphi:(a, b)_{\mathbb{Z}} \rightarrow \mathbb{R}, \xi_{a}, \xi_{b} \in$ $C^{1}\left(\mathbb{R}_{0}^{+}\right)$are initial-boundary conditions, and $\mathbb{R}_{0}^{+}:=[0,+\infty)$.

Given the fact that (36) can be interpreted as a vector ODE, we can rewrite it as

$$
\begin{aligned}
& \mathbf{u}^{\prime}(t)=\mathbf{g}(t, \mathbf{u}(t)), \quad t \in \mathbb{R}_{0}^{+}, \\
& \mathbf{u}(0)=\widetilde{\mathbf{u}},
\end{aligned}
$$

where $\mathbf{u}: \mathbb{R}_{0}^{+} \rightarrow \mathbb{R}^{N}, \mathbf{g}: \mathbb{R}_{0}^{+} \times \mathbb{R}^{N} \rightarrow \mathbb{R}^{N}$ is continuous and $\widetilde{\mathbf{u}} \in \mathbb{R}^{N}$

Naturally, we use the well-known result of Picard and Lindelöf to get the local existence for the initial value problem (37) (see [20, Theorem 8.13]).

Theorem 17. Assume that $\mathbf{g}$ is continuous on the rectangle

$$
Q=\left\{(t, \mathbf{u}) \in \mathbb{R}_{0}^{+} \times \mathbb{R}^{N}: 0 \leq t \leq \alpha,\|\mathbf{u}-\widetilde{\mathbf{u}}\| \leq \beta\right\}
$$

and satisfies the Lipschitz condition on $Q$; that is, there exists $L>0$ such that, for all $\left(t_{1}, \mathbf{u}_{1}\right),\left(t_{2}, \mathbf{u}_{2}\right) \in Q$

$$
\left\|\mathbf{g}\left(t_{1}, \mathbf{u}_{1}\right)-\mathbf{g}\left(t_{2}, \mathbf{u}_{2}\right)\right\| \leq L\left\|\mathbf{u}_{1}-\mathbf{u}_{2}\right\|
$$

holds. Then there exists $\eta>0$ such that (37) has a unique solution $\mathbf{u}$ defined on $[0, \eta]$.

We apply Theorem 17 to get the local existence for the semidiscrete reaction-diffusion equation (36). We use the following two assumptions:

$\left(C_{\text {cont }}\right)$ Let $f(x, t, u)$ be continuous in $(t, u) \in \mathbb{R}_{0}^{+} \times \mathbb{R}$ for all $x \in(a, b)_{\mathbb{Z}}$.

$\left(C_{\text {lip }}\right)$ Let $f(x, t, u)$ be locally Lipschitz with respect to $u$ on $(a, b)_{\mathbb{Z}} \times \mathbb{R}_{0}^{+} \times \mathbb{R}$; that is, for all $x_{0} \in(a, b)_{\mathbb{Z}}, t_{0} \in \mathbb{R}_{0}^{+}$, and $u_{0} \in \mathbb{R}$ there exist $\alpha, \beta>0$ and

$$
\begin{gathered}
Q\left(x_{0}, t_{0}, u_{0}\right)=\left\{\left(x_{0}, t, u\right) \in(a, b)_{\mathbb{Z}} \times \mathbb{R}_{0}^{+}\right. \\
\left.\times \mathbb{R}:\left|t-t_{0}\right| \leq \alpha,\left|u-u_{0}\right| \leq \beta\right\}
\end{gathered}
$$

and $L>0$ such that for all $\left(x_{0}, t_{1}, u_{1}\right),\left(x_{0}, t_{2}, u_{2}\right) \in Q$ there is

$$
\left|f\left(x_{0}, t_{1}, u_{1}\right)-f\left(x_{0}, t_{2}, u_{2}\right)\right| \leq L\left|u_{1}-u_{2}\right| .
$$

Theorem 18. Let $f$ satisfy $\left(C_{\text {cont }}\right)$ and $\left(C_{\text {lip }}\right)$. Then there exists $\eta>0$ such that (36) has a unique solution defined on $[a, b]_{\mathbb{Z}} \times$ $[0, \eta]$.

Proof. Since the space variable $x$ is from a finite set $(a, b)_{\mathbb{Z}}$ problem (36) corresponds to the following vector ODE:

$$
\begin{aligned}
& \underbrace{\left(\begin{array}{c}
u(a+1, t) \\
u(a+2, t) \\
u \\
u(b-2, t) \\
u(b-1, t)
\end{array}\right)}_{\mathbf{u}^{\prime}(t)}
\end{aligned}
$$

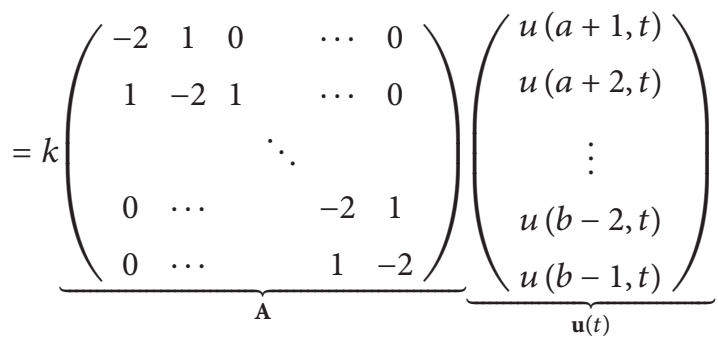

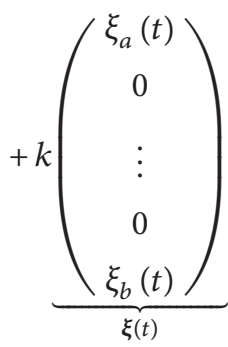

$$
+\underbrace{\left(\begin{array}{c}
f(a+1, t, u(a+1, t)) \\
f(a+2, t, u(a+2, t)) \\
\vdots \\
f(b-2, t, u(b-2, t)) \\
f(b-1, t, u(b-1, t))
\end{array}\right)}_{\mathbf{f}(t, \mathbf{u}(t))}
$$

coupled with the initial condition

$$
\underbrace{\left(\begin{array}{c}
u(a+1,0) \\
u(a+2,0) \\
\vdots \\
u(b-1,0)
\end{array}\right)}_{\mathbf{u}(0)}=\underbrace{\left(\begin{array}{c}
\varphi(a+1) \\
\varphi(a+2) \\
\vdots \\
\varphi(b-1)
\end{array}\right)}_{\varphi}
$$

Thus, problem (36) can be rewritten in the vector form as follows:

$$
\begin{aligned}
& \mathbf{u}^{\prime}(t)=k \mathbf{A u}(t)+k \xi(t)+\mathbf{f}(t, \mathbf{u}(t)), \quad t \in \mathbb{R}_{0}^{+}, \\
& \mathbf{u}(0)=\boldsymbol{\varphi} .
\end{aligned}
$$


Assumptions $\left(C_{\text {cont }}\right)$ and $\left(C_{\text {lip }}\right)$ yield that the nonlinear function $\mathbf{f}$ is continuous and satisfies Lipschitz condition with respect to $\mathbf{u}$ on some rectangle $Q$. Since the term $\mathbf{A u}$ is linear and therefore Lipschitz with respect to $\mathbf{u}$ and $\xi \in C^{1}\left(\mathbb{R}_{0}^{+}\right)$the assumptions of Theorem 17 are satisfied. Consequently, there exists $\eta>0$ such that (44) has a unique solution on $[0, \eta]$.

Remark 19. If we assume only $\left(C_{\text {cont }}\right)$ then we can apply the Peano theorem [20, Theorem 8.27] instead of Theorem 17 to get the local existence of solutions of (36) which need not be unique.

\section{Semidiscrete Reaction-Diffusion Equation: Maximum Principles}

Having the local existence and uniqueness we focus on the maximum principles for (36). In the following analysis we approximate the solution of (36) by the solutions of the discrete problem (8) which arises from (36) by the explicit (Euler) discretization of the time variable.

First, we define the Euler polygon (see [21, I.7]).

Definition 20. Let $h>0$ be a discretization step. Consider the initial value problem (37) on the interval $[0, T]$ where $T=n h$, $n \in \mathbb{N}$. Define the subdivision of interval $[0, T]$ as the set of points $t_{i}=i h, i=0,1, \ldots, n$, and for $i=0,1, \ldots, n-1$ define

$$
\begin{aligned}
\mathbf{y}_{i+1} & =\mathbf{y}_{i}+h \mathbf{g}\left(t_{i}, \mathbf{y}_{i}\right), \\
\mathbf{y}_{0} & =\mathbf{u}(0)=\widetilde{\mathbf{u}} .
\end{aligned}
$$

Then the continuous function $\mathbf{y}_{(h)}:[0, T] \rightarrow \mathbb{R}^{N}$ defined by

$$
\mathbf{y}_{(h)}(t)=\mathbf{y}_{i}+\left(t-t_{i}\right) \mathbf{g}\left(t_{i}, \mathbf{y}_{i}\right), \quad t_{i} \leq t \leq t_{i+1},
$$

is called Euler polygon.

The following statement sums up the convergence of Euler method (see [21, I.7, Theorem 7.3 and I.9, page 54]).

Theorem 21. Let $T>0$ and let $\mathbf{g}$ be continuous, satisfying Lipschitz condition on

$$
Q=\left\{(t, \mathbf{u}) \in \mathbb{R}_{0}^{+} \times \mathbb{R}^{N}: 0 \leq t \leq T,\|\mathbf{u}-\widetilde{\mathbf{u}}\| \leq \beta\right\},
$$

and let $\|\mathbf{g}\|$ be bounded by a constant $A>0$ on $Q$. If $T \leq \beta / A$ then the following hold:

(a) for $h \rightarrow 0+$ the Euler polygons $\mathbf{y}_{(h)}(t)$ converge uniformly to a continuous function $\vartheta(t)$ on $[0, T]$,

(b) $\vartheta \in C^{1}(0, T)$ and it is the unique solution of (37) on $[0, T]$.

We define the bounds of initial-boundary conditions similarly as in the discrete problem:

$$
\begin{aligned}
M_{T}:= & \max _{x \in(a, b)_{\mathbb{Z}}, t \in[0, T]}\left\{\varphi(x), \xi_{a}(t), \xi_{b}(t)\right\}, \\
m_{T}: & =\min _{x \in(a, b)_{\mathbb{Z}}, t \in[0, T]}\left\{\varphi(x), \xi_{a}(t), \xi_{b}(t)\right\} .
\end{aligned}
$$

Before we state the weak maximum principle we describe the connection between the discretization of (36) and the assumption $(D)$.

Lemma 22. Let $T \in \mathbb{R}_{0}^{+}$and let $f$ satisfy $\left(C_{\text {cont }}\right),\left(C_{\text {lip }}\right)$, and $\left(C_{\text {sign }}\right) f\left(x, t, M_{T}\right) \leq 0 \leq f\left(x, t, m_{T}\right)$ for all $x \in(a, b)_{\mathbb{Z}}, t \in$ $[0, T]$.

Then there exists $H>0$ such that for all $h \in(0, H)$

$$
\frac{2 h k-1}{h}\left(u-m_{T}\right) \leq f(x, t, u) \leq \frac{2 h k-1}{h}\left(u-M_{T}\right)
$$

holds for all $x \in(a, b)_{\mathbb{Z}}, t \in[0, T]$, and $u \in\left[m_{T}, M_{T}\right]$.

Proof. We prove the latter inequality in (49) by contradiction. The former inequality can be proved in the same way. Let us assume that for all $H>0$ there exist $h \in(0, H), x_{h} \in(a, b)_{\mathbb{Z}}$, $t_{h} \in[0, T]$, and $u_{h} \in\left[m_{T}, M_{T}\right]$ such that

$$
f\left(x_{h}, t_{h}, u_{h}\right)>\frac{2 h k-1}{h}\left(u_{h}-M_{T}\right) .
$$

Therefore, there exist sequences $\left\{h_{m}\right\}_{m=1}^{\infty}$ and $\left\{\left(x_{m}, t_{m}\right.\right.$, $\left.\left.u_{m}\right)\right\}_{m=1}^{\infty}$ (we denote $\left.x_{m}:=x_{h_{m}}, t_{m}:=t_{h_{m}}, u_{m}:=u_{h_{m}}\right)$ such that

$$
\begin{gathered}
h_{m} \longrightarrow 0+ \\
f\left(x_{m}, t_{m}, u_{m}\right)>\frac{2 h_{m} k-1}{h_{m}}\left(u_{m}-M_{T}\right) .
\end{gathered}
$$

First, we observe that if $u_{m}=M_{T}$ for some $m \in \mathbb{N}$ we get a contradiction with $\left(C_{\text {sign }}\right)$. Thus, we can assume that $u_{m}<$ $M_{T}$ for all $m \in \mathbb{N}$.

Now we have to distinguish between two cases.

(i) If there does not exist any subsequence $\left\{u_{m_{l}}\right\}_{l=1}^{\infty} \subset$ $\left\{u_{m}\right\}_{m=1}^{\infty}$ such that $u_{m_{l}} \rightarrow M_{T}$ then the right-hand side of inequality in (51) goes to infinity. Hence, from (51) $f\left(x_{m}, t_{m}, u_{m}\right)$ also goes to infinity. This yields a contradiction with $\left(C_{\text {cont }}\right)$, which implies boundedness of the function $f$ on $(a, b)_{\mathbb{Z}} \times[0, T] \times$ $\left[m_{T}, M_{T}\right]$.

(ii) Let there exist a subsequence $\left\{u_{m_{l}}\right\}_{l=1}^{\infty} \subset\left\{u_{m}\right\}_{m=1}^{\infty}$ such that $u_{m_{l}} \rightarrow M_{T}$. We show that we get a contradiction with $\left(C_{\text {lip }}\right)$ in this case. Since the interval $(a, b)_{\mathbb{Z}}$ is bounded there exists a convergent subsequence $\left\{x_{m_{l}}\right\}_{l=1}^{\infty} \subset\left\{x_{m}\right\}_{m=1}^{\infty}$ such that $x_{m_{l}} \rightarrow \tilde{x}$. Analogically, since $[0, T]$ is bounded there also exists a convergent subsequence $\left\{t_{m_{l}}\right\}_{l=1}^{\infty} \subset\left\{t_{m}\right\}_{m=1}^{\infty}$ such that $t_{m_{l}} \rightarrow \tilde{t}$. Let $\alpha, \beta>0$, and $L>0$ be arbitrary. Then we can find $\hat{l} \in \mathbb{N}$ sufficiently large such that

$$
\begin{aligned}
\frac{1-2 h_{m_{\tilde{l}}} k}{h_{m_{\hat{\imath}}}} & \geq L, \\
M_{T}-u_{m_{\hat{\imath}}} & \leq \beta, \\
x_{m_{\hat{\imath}}} & =\tilde{x}, \\
\left|t_{m_{\tilde{\imath}}}-\tilde{t}\right| & <\alpha .
\end{aligned}
$$


If we put $\widehat{x}:=x_{m_{\widehat{\imath}}}, \widehat{t}:=t_{m_{\hat{\imath}}}, \widehat{u}:=u_{m_{\hat{\imath}}}$, and $\widetilde{u}:=M_{T}$ and $Q(\tilde{x}, \widetilde{t}, \widetilde{u})$ is the rectangle from assumption $\left(C_{\text {lip }}\right)$ with given $\alpha>0$ and $\beta>0$ then $(\widehat{x}, \widehat{t}, \widehat{u}),(\widetilde{x}, \widetilde{t}, \widetilde{u}) \epsilon$ $Q(\widetilde{x}, \tilde{t}, \widetilde{u})$. Now from (51), (52), and $\left(C_{\text {sign }}\right)$ we can estimate

$$
\begin{aligned}
L|\widetilde{u}-\widehat{u}| & \leq \frac{1-2 h_{m_{\hat{\imath}}} k}{h_{m_{\hat{\imath}}}}\left(M_{T}-\widehat{u}\right)<f(\widehat{x}, \widehat{t}, \widehat{u}) \\
& \leq f(\widehat{x}, \widehat{t}, \widehat{u})-f\left(\tilde{x}, \widetilde{t}, M_{T}\right) \\
& =f(\widehat{x}, \widehat{t}, \widehat{u})-f(\tilde{x}, \tilde{t}, \widetilde{u}) \\
& \leq|f(\widehat{x}, \widehat{t}, \widehat{u})-f(\tilde{x}, \widetilde{t}, \widetilde{u})|,
\end{aligned}
$$

a contradiction with $\left(C_{\text {lip }}\right)$.

Remark 23. The assumption $\left(C_{\text {sign }}\right)$ defines the forbidden area for a reaction function $f(x, t, \cdot)$ in the same way as $(D)$. However, this area is reduced to a pair of half-lines. Let us notice that it is the limit case of forbidden areas for the discrete case if $h \rightarrow 0+$ (see Remark 5 and Figure 1). Moreover, it is equivalent to the assumption for classical PDEs; see (5).

Theorem 24. Let $T>0$ be arbitrary, let $f$ satisfy $\left(C_{\text {cont }}\right),\left(C_{\text {lip }}\right)$, and $\left(C_{\text {sign }}\right)$, and let $u$ be a solution of (36) defined on $[a, b]_{\mathbb{Z}} \times$ $[0, T]$. Then

$$
m_{T} \leq u(x, t) \leq M_{T}
$$

holds for all $x \in(a, b)_{\mathbb{Z}}, t \in[0, T]$.

Proof. We prove that for all $x \in(a, b)_{\mathbb{Z}}, t \in[0, T]$ there is $u(x, t) \leq M_{T}$. The first inequality in (54) can be proved similarly. Let us assume by contradiction that there exist $x_{c} \in$ $(a, b)_{\mathbb{Z}}$ and $t_{c} \in(0, T]$ such that

$$
u\left(x_{c}, t_{c}\right)>M_{\mathrm{T}}
$$

From the continuity of the solution $u$ there exist $x_{0} \in(a, b)_{\mathbb{Z}}$ and $t_{0} \in\left[0, t_{c}\right)$ such that
(a) $u(x, t) \leq M_{T}$ for all $x \in(a, b)_{\mathbb{Z}}$ and $t \in\left[0, t_{0}\right]$,
(b) $u\left(x_{0}, t_{0}\right)=M_{T}$,
(c) there exists $\delta>0$ such that$$
u\left(x_{0}, t\right)>M_{T} \text { on }\left(t_{0}, t_{0}+\delta\right) .
$$

Let us analyze the new initial-boundary value problem (36) with the initial condition $u\left(x, t_{0}\right)$ at time $t_{0}$. Let us understand this problem as the initial value problem for the vector ODE (44) with the initial condition at time $t_{0}$.

From $\left(C_{\text {cont }}\right),\left(C_{\text {lip }}\right)$ we know that $\mathbf{f}(t, \mathbf{u})$ is continuous and Lipschitz on some rectangle $Q$. From $\left(C_{\text {cont }}\right)$ we also get that $\mathbf{f}$ is bounded by some constant $A>0$ on $Q$. Therefore, Theorem 21 implies that for sufficiently small discretization steps $h>0$ and for sufficiently small interval $\left[t_{0}, t_{0}+\varepsilon\right]$ the Euler polygons $\mathbf{y}_{(h)}(t)$ converge uniformly to the unique solution $\mathbf{u}(t)$ on $\left[t_{0}, t_{0}+\varepsilon\right]$.

Notice that the node points of Euler polygons $\mathbf{y}_{(h)}(t)$ are the solutions of (8). From $\left(C_{\text {cont }}\right),\left(C_{\text {lip }}\right)$, and $\left(C_{\text {sign }}\right)$ and from Lemma 22 the assumption $(D)$ is satisfied (recall that $h$ is sufficiently small) and therefore, from Theorem 9

$$
y_{(h)}(x, t) \leq M_{T} \text { on }\left[t_{0}, t_{0}+\varepsilon\right], \forall x \in(a, b)_{\mathbb{Z}} .
$$

But if $\mathbf{y}_{(h)}(t)$ converge uniformly to $\mathbf{u}(t)$ and $y_{(h)}(x, t) \leq M_{T}$ on $\left[t_{0}, t_{0}+\min \{\delta, \varepsilon\}\right]$ for all $x \in(a, b)_{\mathbb{Z}}$ then there has to be

$$
\begin{aligned}
& u(x, t) \leq M_{T} \\
& \quad \text { on }\left[t_{0}, t_{0}+\min \{\delta, \varepsilon\}\right], \forall x \in(a, b)_{\mathbb{Z}},
\end{aligned}
$$

a contradiction with (56).

If the assumption $\left(C_{\text {sign }}\right)$ is not satisfied but the nonlinear function $f$ satisfies the following:

$\left(C_{\text {sign }}^{\prime}\right)$ Let $T>0$ be arbitrary and let there exist $S \geq M_{T}$ and $R \leq m_{T}$ such that

$$
f(x, t, S) \leq 0 \leq f(x, t, R)
$$

for all $x \in(a, b)_{\mathbb{Z}}, t \in[0, T]$,

then we can state the following generalized weak maximum principle.

Theorem 25. Let $T>0$ be arbitrary, let $f$ satisfy $\left(C_{\text {cont }}\right),\left(C_{\text {lip }}\right)$, and $\left(C_{\text {sign }}^{\prime}\right)$, and let $u$ be a solution of (36) defined on $[a, b]_{\mathbb{Z}} \times$ $[0, T]$. Then

$$
R \leq u(x, t) \leq S
$$

holds for all $x \in(a, b)_{\mathbb{Z}}, t \in[0, T]$.

Proof. The statement can be proved in the similar way as Lemma 22 and Theorem 24.

As in the previous sections we want to establish the strong maximum principle. First, we recall the well-known Grönwall's inequality (see, e.g., [20, Corollary 8.62]).

Lemma 26. Let $\beta, u:[r, s] \rightarrow \mathbb{R}$ be continuous functions and let $u$ be differentiable on $(r, s)$. If

$$
u^{\prime}(t) \leq \beta(t) u(t) \quad \text { for } t \in(r, s)
$$

then

$$
u(t) \leq u(r) e^{\int_{r}^{t} \beta(\tau) d \tau} \quad \text { for } t \in[r, s] .
$$

Further, we need the following auxiliary lemma.

Lemma 27. Let $T>0$ be arbitrary, let $f$ satisfy $\left(C_{\text {cont }}\right),\left(C_{\text {lip }}\right)$, and $\left(C_{\text {sign }}\right)$, and let $u$ be a solution of (36) defined on $[a, b]_{\mathbb{Z}} \times$ $[0, T]$. If $u\left(x_{0}, t_{0}\right)=M_{T}\left(\right.$ or $\left.u\left(x_{0}, t_{0}\right)=m_{T}\right)$ for some $x_{0} \in$ $(a, b)_{\mathbb{Z}}$ and $t_{0} \in(0, T]$ then

$$
\begin{gathered}
u\left(x_{0}, t\right)=M_{T} \\
\left(\operatorname{or} u\left(x_{0}, t\right)=m_{T}\right)
\end{gathered}
$$


Proof. We prove the statement with $M_{T}$. The case with $m_{T}$ is similar. First, the weak maximum principle (Theorem 24) holds; that is, $u(x, t) \leq M_{T}$ for all $x \in[a, b]_{\mathbb{Z}}$ and $t \in[0, T]$. Suppose by contradiction that there exists $t_{c} \in\left(0, t_{0}\right]$ such that

$$
\begin{gathered}
u\left(x_{0}, t_{c}\right)=M_{T}, \\
u\left(x_{0}, t\right)<M_{T}
\end{gathered}
$$

$$
\text { on }\left[t_{c}-\varepsilon, t_{c}\right) \text {. }
$$

Without loss of generality let $\varepsilon>0$ be sufficiently small so that the function $f\left(x_{0}, t, u\right)$ is uniformly Lipschitz in $u$ on $\left[t_{c}-\right.$ $\varepsilon, t_{c}$ ) with Lipschitz constant $L>0$ (follows from $\left(C_{\text {lip }}\right)$ ). With the help of these facts and also from $\left(C_{\text {sign }}\right)$ we can estimate

$$
\begin{aligned}
u_{t}\left(x_{0}, t\right) & \\
= & k\left(u\left(x_{0}-1, t\right)-2 u\left(x_{0}, t\right)+u\left(x_{0}+1, t\right)\right) \\
& +f\left(x_{0}, t, u\left(x_{0}, t\right)\right) \\
\leq & k\left(2 M_{T}-2 u\left(x_{0}, t\right)\right)+f\left(x_{0}, t, u\left(x_{0}, t\right)\right) \\
& \quad-f\left(x_{0}, t, M_{T}\right)+\underbrace{f\left(x_{0}, t, M_{T}\right)}_{\leq 0} \\
\leq & k\left(2 M_{T}-2 u\left(x_{0}, t\right)\right) \\
& +\left|f\left(x_{0}, t, u\left(x_{0}, t\right)\right)-f\left(x_{0}, t, M_{T}\right)\right| \\
\leq & k\left(2 M_{T}-2 u\left(x_{0}, t\right)\right)+L\left|u\left(x_{0}, t\right)-M_{T}\right| \\
= & k\left(2 M_{T}-2 u\left(x_{0}, t\right)\right)+L\left(M_{T}-u\left(x_{0}, t\right)\right) \\
= & -(2 k+L)\left(u\left(x_{0}, t\right)-M_{T}\right),
\end{aligned}
$$

for all $t \in\left(t_{c}-\varepsilon, t_{c}\right)$. If we denote $\alpha:=-(2 k+L)$ then the function $u\left(x_{0}, t\right)$ satisfies $u_{t}\left(x_{0}, t\right) \leq \alpha\left(u\left(x_{0}, t\right)-M_{T}\right)$ on $\left(t_{c}-\right.$ $\left.\varepsilon, t_{c}\right)$. If we substitute $v(t):=u\left(x_{0}, t\right)-M_{T}$ then the function $v$ satisfies the following differential inequality:

$$
v^{\prime}(t) \leq \alpha v(t) .
$$

Therefore, Grönwall's inequality (Lemma 26) implies that

$$
v(t) \leq v\left(t_{c}-\varepsilon\right) e^{\alpha\left(t-t_{c}+\varepsilon\right)}
$$

and hence

$$
\begin{aligned}
u\left(x_{0}, t\right) & \leq M_{T}-\left(M_{T}-u\left(x_{0}, t_{c}-\varepsilon\right)\right) e^{\alpha\left(t-t_{c}+\varepsilon\right)} \\
& <M_{T}
\end{aligned}
$$

on $\left[t_{c}-\varepsilon, t_{c}\right]$, a contradiction. ately.

The strong maximum principle for (36) follows immedi-

Theorem 28. Let $T>0$ be arbitrary, let $f$ satisfy $\left(C_{\text {cont }}\right)$, $\left(C_{\text {lip }}\right)$, and $\left(C_{\text {sign }}\right)$, and let $u$ be a solution of (36) defined on $[a, b]_{\mathbb{Z}} \times[0, T]$. If $u\left(x_{0}, t_{0}\right)=M_{T}\left(\right.$ or $\left.u\left(x_{0}, t_{0}\right)=m_{T}\right)$ for some $x_{0} \in(a, b)_{\mathbb{Z}}$ and $t_{0} \in(0, T]$ then

$$
\begin{aligned}
& u(x, t)=M_{T} \\
&(\operatorname{or} u(x, t)\left.=m_{T}\right) \\
& \forall x \in[a, b]_{\mathbb{Z}}, t \in\left[0, t_{0}\right] .
\end{aligned}
$$

Proof. Lemma 27 yields that $u\left(x_{0}, t\right)=M_{T}$ for all $t \in\left[0, t_{0}\right]$. If $u\left(x_{0}-1, t_{c}\right)<M_{T}\left(\right.$ or $\left.u\left(x_{0}+1, t_{c}\right)<M_{T}\right)$ at some $t_{c} \in\left[0, t_{0}\right]$ then applying $\left(C_{\text {sign }}\right)$ the following has to be satisfied:

$$
\begin{aligned}
u_{t}\left(x_{0}, t_{c}\right) & \\
= & k\left(u\left(x_{0}-1, t_{c}\right)-2 u\left(x_{0}, t_{c}\right)+u\left(x_{0}+1, t_{c}\right)\right) \\
& +f\left(x_{0}, t_{c}, u\left(x_{0}, t_{c}\right)\right) \\
< & k\left(2 M_{T}-2 M_{T}\right)+f\left(x_{0}, t_{c}, M_{T}\right) \leq 0,
\end{aligned}
$$

a contradiction with the fact that the function $u\left(x_{0}, t\right)$ is constant and equal to $M_{T}$ on $\left[0, t_{0}\right]$. Therefore, functions $u\left(x_{0}-1, t\right)$ and $u\left(x_{0}+1, t\right)$ are also constant and equal to $M_{T}$ on $\left[0, t_{0}\right]$. Then we can continue inductively in $x$ to the boundary points $x=a$ or $x=b$. The case with $m_{T}$ is similar.

\section{Semidiscrete Reaction-Diffusion Equation: Global Existence}

In this section we combine the local existence and uniqueness and the maximum principle to obtain the global existence of solution of (36).

Once again, we use known results from the theory of ordinary differential equations. First, we define the maximal interval of existence (see [20, Definition 8.31]).

Definition 29. Let $\mathbf{g}$ be continuous and let $\mathbf{u}$ be a solution of $(37)$ defined on $[0, \eta)$. Then one says $[0, \eta)$ is a maximal interval of existence for $\mathbf{u}$ if there does not exist an $\eta_{1}>\eta$ and a solution $\mathbf{w}$ defined on $\left[0, \eta_{1}\right)$ such that $\mathbf{u}(t)=\mathbf{w}(t)$ for $t \in[0, \eta)$.

In the following we apply the extendability theorem (see [20, Theorem 8.33]).

Theorem 30. Let $\mathbf{g}$ be continuous and let $\mathbf{u}$ be a solution of (37) defined on $[0, \omega)$. Then $u$ can be extended to a maximal interval of existence $[0, \eta), 0<\eta \leq \infty$. Furthermore, there is either

$$
\begin{gathered}
\eta=\infty \\
\text { or }\|\mathbf{u}(t)\| \stackrel{t \rightarrow \eta^{-}}{\longrightarrow} \infty .
\end{gathered}
$$

This theorem enables us to conclude with the global existence for (36). 
Theorem 31. Let $f$ satisfy $\left(C_{\text {cont }}\right),\left(C_{\text {lip }}\right)$, and $\left(C_{\text {sign }}\right)$ for all $T>$ 0 . Then (36) has a unique solution defined on $[a, b]_{\mathbb{Z}} \times \mathbb{R}_{0}^{+}$which satisfies

$$
\begin{gathered}
\inf _{x \in(a, b)_{\mathbb{Z}}, t \in \mathbb{R}_{0}^{+}}\left\{\varphi(x), \xi_{a}(t), \xi_{b}(t)\right\} \leq u(x, t) \\
\leq \sup _{x \in(a, b)_{\mathbb{Z}}, t \in \mathbb{R}_{0}^{+}}\left\{\varphi(x), \xi_{a}(t), \xi_{b}(t)\right\},
\end{gathered}
$$

for all $(x, t) \in[a, b]_{\mathbb{Z}} \times \mathbb{R}_{0}^{+}$.

Proof. Let us understand problem (36) as the initial value problem for the vector ODE (44). From Theorem 18 there exists a uniquely determined local solution $\mathbf{u}(t)$. From Theorem 30 the solution can be extended to a maximal interval of existence $[0, \eta)$ which is open from right and either

$$
\begin{gathered}
\eta=\infty \\
\text { or }\|\mathbf{u}(t)\| \stackrel{t \rightarrow \eta-}{\longrightarrow} \infty .
\end{gathered}
$$

If $\|\mathbf{u}(t)\| \rightarrow \infty$ for $t \rightarrow \eta$ - then for all $K>0$ there have to exist $x_{K} \in(a, b)_{\mathbb{Z}}$ and $t_{K} \in[0, \eta)$ such that

$$
\left|u\left(x_{K}, t_{K}\right)\right|>K \text {. }
$$

If we put $K:=\max \left\{\left|m_{\eta}\right|,\left|M_{\eta}\right|\right\}<\infty\left(\xi_{a}, \xi_{b}\right.$ are $C^{1}$ functions on $\mathbb{R}_{0}^{+}$and therefore bounded on $\left.[0, \eta]\right)$ then

$$
\left|u\left(x_{K}, t_{K}\right)\right|>\max \left\{\left|m_{\eta}\right|,\left|M_{\eta}\right|\right\},
$$

a contradiction with the maximum principle in Theorem 24 (which holds thanks to $\left(C_{\text {cont }}\right),\left(C_{\text {lip }}\right)$, and $\left(C_{\text {sign }}\right)$ ).

Therefore, there has to be $\eta=\infty$; that is, the solution $\mathbf{u}(t)$ is defined on the interval $[0, \infty)$ and from $\left(C_{\text {lip }}\right)$ it has to be unique.

Remark 32. All nonlinear functions $f$ listed in Example 12 can be considered in Theorems 24 and 31. However, it is worth noting that we have additional assumptions on the nonlinearity in the semidiscrete case (conditions $\left(C_{\text {cont }}\right)$ and $\left.\left(C_{\text {lip }}\right)\right)$. Thus, for non-Lipschitz functions (e.g.,

$$
f(x, t, u)= \begin{cases}-|u|^{p-1} u, & u \neq 0, \\ 0, & u=0\end{cases}
$$

with $p \in[0,1)$ ), we only get the maximum principles in discrete case. On the other hand, in discrete case, the validity of maximum principle depends strongly on the interaction between the discretization step $h$ and the nonlinearity $f$ (see (12); we illustrate this dependence in detail in Section 8).

Let us finish with the two corollaries that are immediate consequences of Theorems 24 and 31.

Corollary 33. Assume that $\xi_{a}, \xi_{b}$ are bounded. Let $f$ satisfy $\left(C_{\text {cont }}\right),\left(C_{\text {lip }}\right)$, and $\left(C_{\text {sign }}\right)$ for all $T>0$. Then the unique solution $u$ of (36) is bounded.
Corollary 34. Assume that $\varphi, \xi_{a}, \xi_{b}$ are nonnegative. Let $f$ satisfy $\left(C_{\text {cont }}\right),\left(C_{\text {lip }}\right)$, and $\left(C_{\text {sign }}\right)$ for all $T>0$. Then the unique solution $u$ of (36) is nonnegative.

\section{Application: Discrete and Semidiscrete Nagumo Equation}

In this section we apply the results of this paper to the most common nonlinearity occurring in the connection with the reaction-diffusion equation, the bistable/double-well nonlinearity. For simplicity, we consider only the symmetric case and use interval $[-1,1]$ so that our arguments for positive values can be directly reproduced for the negative ones; that is, we study

$$
f(x, t, u)=\lambda u\left(1-u^{2}\right), \quad \lambda \in \mathbb{R} .
$$

Throughout this section we assume that the initial-boundary conditions $\varphi, \xi_{a}, \xi_{b}$ are such that $m_{T}=-1$ and $M_{T}=1$ (or possibly $m_{T} \geq-1$ and $\left.M_{T} \leq 1\right)$ for all $T>0$.

Starting with the semidiscrete case (36), we observe that $f$ is continuous and locally Lipschitz continuous and satisfies $f(x, t,-1)=0=f(x, t, 1)$. Consequently, for any given $\lambda \epsilon$ $\mathbb{R}$ we can apply Theorems 24 and 31 to get that there exists a unique solution of the semidiscrete problem (36) such that $u(x, t) \in[-1,1]$ for all $x \in(a, b)_{\mathbb{Z}}$ and $t \in \mathbb{R}_{0}^{+}$.

We encounter a more interesting situation if we consider the problem for the discrete Nagumo equation

$$
\begin{aligned}
& \frac{u(x, t+h)-u(x, t)}{h}= k \Delta_{x x}^{2} u(x-1, t) \\
&+\lambda u(x, t)\left(1-u^{2}(x, t)\right), \\
& u(x, 0)= \varphi(x), \quad x \in(a, b)_{\mathbb{Z}}, \\
& u(a, t)=\xi_{a}(t), \quad t \in h \mathbb{N}_{0}, \\
& u(b, t)=\xi_{b}(t), \quad t \in h \mathbb{N}_{0},
\end{aligned}
$$

with $\lambda \in \mathbb{R}, x \in(a, b)_{\mathbb{Z}}, t \in h \mathbb{N}_{0}, h>0, k>0$.

Let us assume first that $\lambda>0$. We observe that $f^{\prime}(1)=$ $-2 \lambda$. Hence the application of Theorem 9 is restricted to cases for which the slope of the dashed line in the forbidden area (see Figure 1) given by $2 k-1 / h$ (see the assumption $(D)$ ) satisfies

$$
\begin{aligned}
2 k-\frac{1}{h} & \leq-2 \lambda, \quad \text { or equivalently } \\
h & \leq \frac{1}{2(k+\lambda)} .
\end{aligned}
$$

Consequently, if $h \leq 1 / 2(k+\lambda)$, we can apply Theorem 9 to get that $u(x, t) \in[-1,1]$ for all $x \in(a, b)_{\mathbb{Z}}, t \in h \mathbb{N}_{0}$ (see Figure 3(a)).

Once $h>1 / 2(k+\lambda)$, Theorem 9 is no longer available and we proceed to Theorem 11. We split our argument into two steps:

(i) Since $f(u)$ is strictly concave on $[0,1]$ and attains a local maximum at $u=1 / \sqrt{3}$, we can for each $h>0$ 


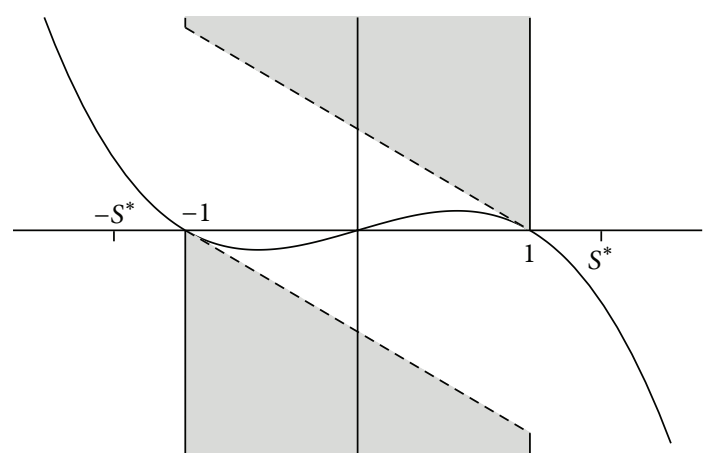

(a) $h \leq 1 / 2(k+\lambda)$

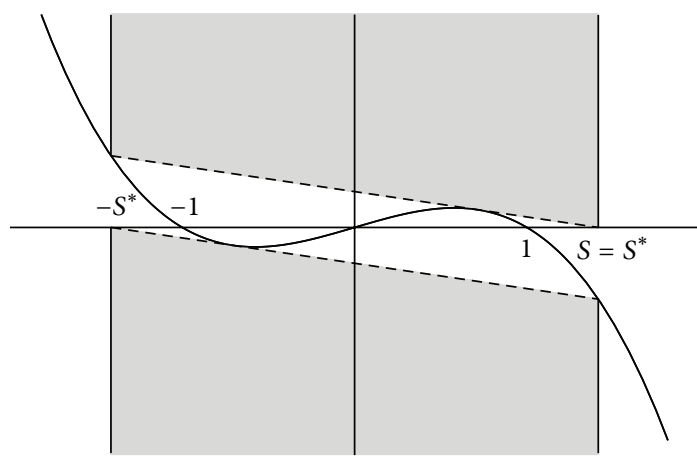

(c) $h=1 /(2 k+\lambda / 2)$ (i.e., $\left.S=S^{*}\right)$

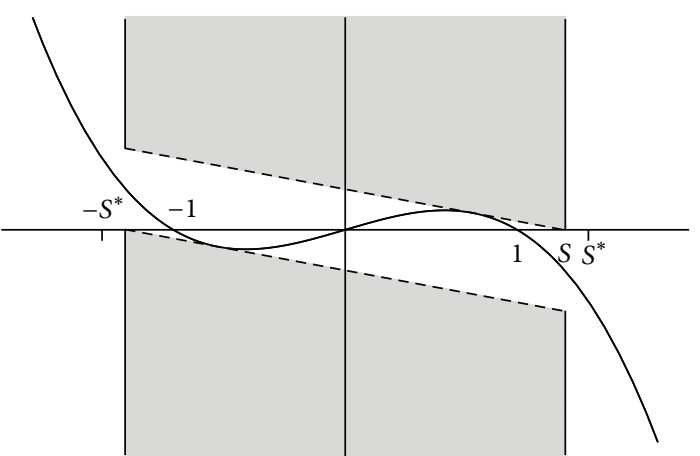

(b) $h \in(1 / 2(k+\lambda), 1 /(2 k+\lambda / 2))$ (i.e., $\left.S \in\left(1, S^{*}\right)\right)$

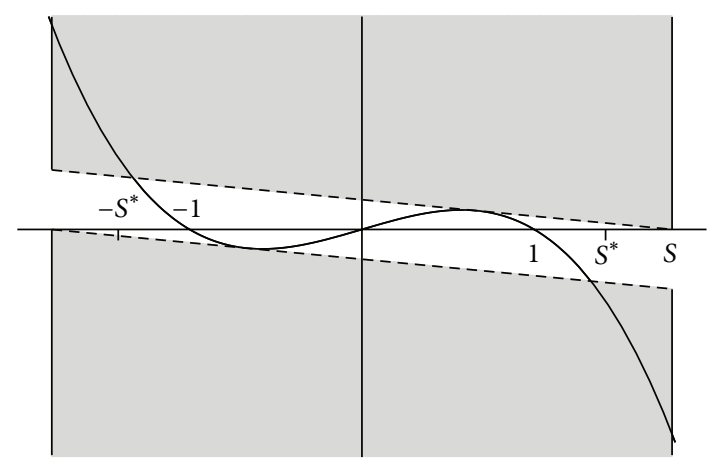

(d) $h>1 /(2 k+\lambda / 2)$ (i.e., $\left.S>S^{*}\right)$

FIGURE 3: Validity of maximum principles for the discrete Nagumo equation (78) with $m_{T}=-1$ and $M_{T}=1$. If $h$ is small enough we can apply Theorem 9; see subplot (a). For $h \in(1 / 2(k+\lambda), 1 /(2 k+\lambda / 2)]$ we can get weaker bounds (83) by the application of Theorem 11 , subplots (b) and (c). If $h$ is greater than $1 /(2 k+\lambda / 2)$ our results cannot be applied; see subplot (d).

such that $2 k-1 / h>-2 \lambda$ (or equivalently $h>1 / 2(k+$ $\lambda)$ ) find a point $T \in(1 / \sqrt{3}, 1)$ such that the slope of a tangent line of $f$ at $T$ is $2 k-1 / h$. One could compute that

$$
T=\sqrt{\frac{1}{3}-\frac{2 k h-1}{3 \lambda h}} .
$$

(ii) Let us denote by $S$ the $x$-intercept of the tangent line of $f$ at $T$. Since the slope of this tangent line is $2 k-1 / h$, we can deduce that

$S=\frac{-2 T^{3}}{1-3 T^{2}}=\frac{2 \lambda h \sqrt{(1 / 3-(2 k h-1) / 3 \lambda h)^{3}}}{1-2 k h}$.

Given the shape of $f(u)$ for $u<0$ (decreasing convexly for $u<-1 / \sqrt{3}$ ), Theorem 11 could be applied once $f(-S)$ lies below or on the tangent line (cf. Figures 2 and 3). We observe the following interesting fact: choosing $S=S^{*}:=\sqrt{2}$ the tangent line of $f$ at $T$ and the function $f$ intersect at the point $[-\sqrt{2}, \lambda \sqrt{2}]$ for each $\lambda>0$ and $k>0$ (see Figure $3(\mathrm{c})$ ).
Consequently, we can apply Theorem 11 whenever $S \leq$ $\sqrt{2}$, which is equivalent to

$$
h \leq \frac{1}{2 k+\lambda / 2} .
$$

If we choose $S>S^{*}$, then we can easily observe that $f(-S)$ lies above the tangent line and therefore Theorem 11 cannot be applied (see Figure 3(d)).

Since we intentionally chose a symmetric $f$, we can repeat the same argument on the lower bound of solutions of (78).

If $\lambda=0$, problem (78) reduces to the linear case and we can trivially apply (12) whenever $h \leq 1 / 2 k$. Finally, if $\lambda<0$, then the assumption $(D)$ is satisfied as long as the line $(2 k-1 / h)(u-1)$ does not intersect for $u<0$ or is tangential to $f(u)=\lambda u\left(1-u^{2}\right)$. One can easily compute that the tangential case occurs if $2 k-1 / h=\lambda / 4$. Therefore, we can apply Theorem 9 whenever $h \leq 1 /(2 k-\lambda / 4)$. If this condition is violated we cannot use Theorem 11 since $f(u)>0$ for $u>1$ and for each $S>1$ the assumption $\left(D^{\prime}\right)$ does not hold.

To sum up, depending on values of $\lambda$ and $h$ we obtain the following bounds for the solution of (78) 


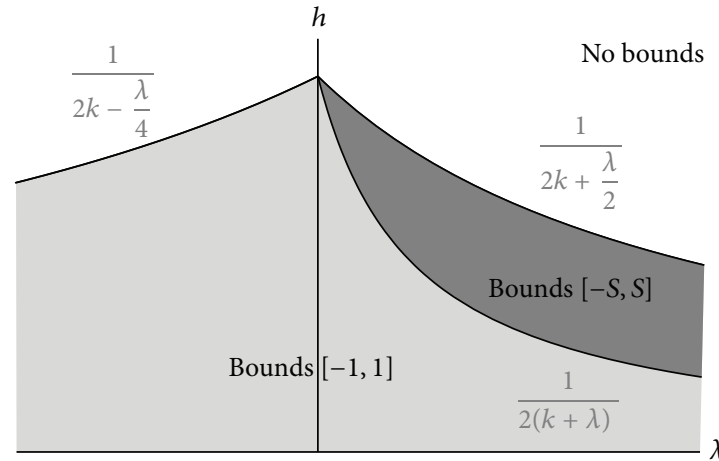

(a)

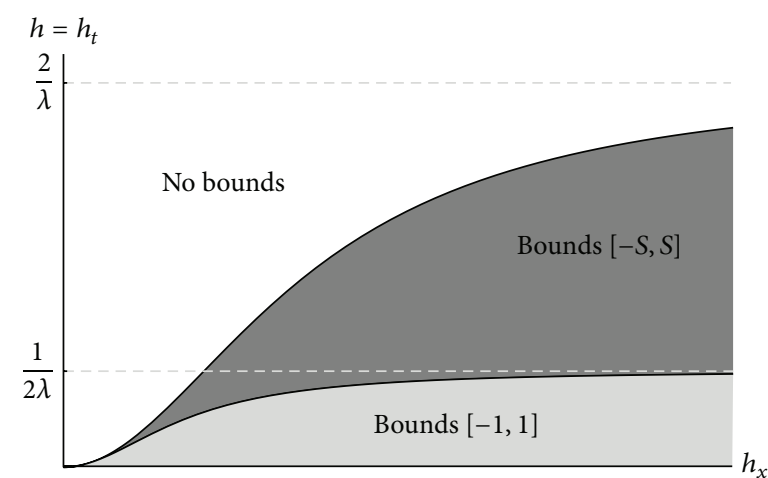

(b)

Figure 4: Bounds of solutions of the discrete Nagumo equation (78) with $m_{T}=-1$ and $M_{T}=1$ and their dependence on the values of $\lambda$ and $h$ (a) and on the values of space and time discretization steps $h_{x}$ and $h=h_{t}$ for a fixed $\lambda>0$ (b). In the light gray area, the bounds follow from Theorem 9. In the dark gray area, the bounds are implied by Theorem 11 and $S$ is given by (81). In the white area we have no bounds on solutions.

$$
\begin{aligned}
& u(x, t)
\end{aligned}
$$

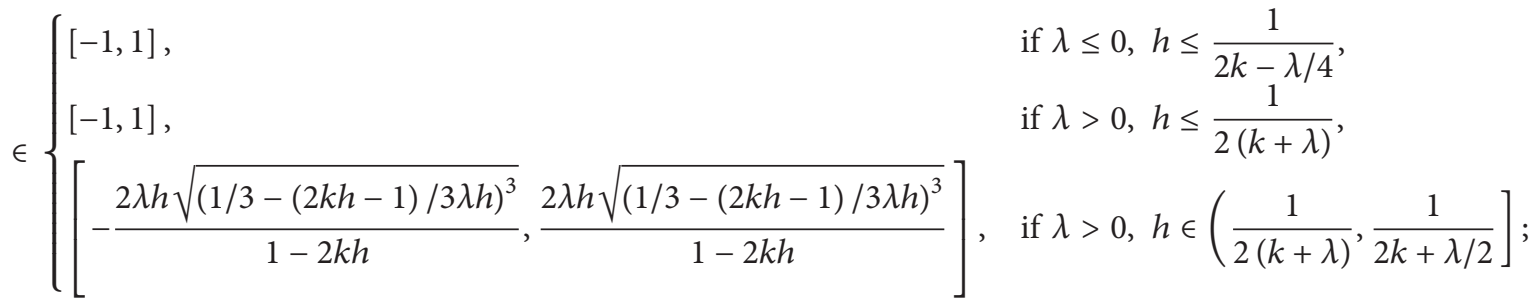

see Figure 4(a) for the illustrative summary of our results in this section.

Interestingly, if we considered general space discretization step $h_{x}>0$ in (78), that is,

$$
\begin{aligned}
& \frac{u(x, t+h)-u(x, t)}{h_{t}} \\
& =k \frac{u\left(x-h_{x}, t\right)-2 u(x, t)+u\left(x+h_{x}, t\right)}{h_{x}^{2}} \\
& \quad+\lambda u(x, t)\left(1-u^{2}(x, t)\right),
\end{aligned}
$$

one could get the same bounds as in (83) by replacing $k$ with $k / h_{x}^{2}$. The dependence of regions of maximum principles' validity on time and space discretization steps $h_{t}$ and $h_{x}$ for $\lambda>0$ is depicted in Figure 4(b) (notice that very small values of $h_{t}$ are necessary for small $h_{x}$ ).

\section{Final Remarks}

In this paper, we studied a priori bounds for solutions of initial-boundary value problems related to discrete and semidiscrete diffusion. Our main motivation for the initialboundary problems was the direct comparison with the classical results (Theorems 2 and 3). However, note that, in the discrete case, the results would be identical if we dealt with an initial problem on $\mathbb{Z}$. On the other hand, in the semidiscrete or classical case, even the solutions of linear diffusion equations are not necessarily bounded (see, e.g., [12]).

Similarly, the ideas of this paper could be easily extended to a general reaction-diffusion-type equation (possibly with nonconstant time steps $h=h(t))$

$$
\begin{aligned}
\frac{u(x, t+h)-u(x, t)}{h}= & a(x, t) u(x-1, t) \\
& +b(x, t) u(x, t) \\
& +c(x, t) u(x+1, t) \\
& +f(x, t, u(x, t)) .
\end{aligned}
$$

For example, the weak maximum principle is then valid if $h \geq$ $-1 / b$ (replacing $h \leq 1 / 2 k$ ) and the following generalization of $(D)$ holds (we assume that $b=-(a+c)<0$ ):

$$
\begin{gathered}
\frac{m_{T}(1-a h-c h)-(1+b h) u}{h} \leq f(x, t, u) \\
\leq \frac{M_{T}(1-a h-c h)-(1+b h) u}{h} .
\end{gathered}
$$




\section{Conflict of Interests}

The authors declare that there is no conflict of interests regarding the publication of this paper.

\section{Acknowledgment}

The authors gratefully acknowledge the support by the Czech Science Foundation, Grant no. GA15-07690S.

\section{References}

[1] J. W. Cahn, "Theory of crystal growth and interface motion in crystalline materials," Acta Metallurgica, vol. 8, no. 8, pp. 554$562,1960$.

[2] J. Bell and C. Cosner, "Threshold behavior and propagation for nonlinear differential-difference systems motivated by modeling myelinated axons," Quarterly of Applied Mathematics, vol. 42, no. 1, pp. 1-14, 1984.

[3] J. P. Keener, "Propagation and its failure in coupled systems of discrete excitable cells," SIAM Journal on Applied Mathematics, vol. 47, no. 3, pp. 556-572, 1987.

[4] S.-N. Chow, "Lattice dynamical systems," in Dynamical Systems, vol. 1822 of Lecture Notes in Mathematics, pp. 1-102, Springer, Berlin, Germany, 2003.

[5] S.-N. Chow, J. Mallet-Paret, and W. Shen, "Traveling waves in lattice dynamical systems," Journal of Differential Equations, vol. 149, no. 2, pp. 248-291, 1998.

[6] B. Zinner, "Existence of traveling wavefront solutions for the discrete Nagumo equation," Journal of Differential Equations, vol. 96, no. 1, pp. 1-27, 1992

[7] L. Xu, L. Zou, Z. Chang, S. Lou, X. Peng, and G. Zhang, "Bifurcation in a discrete competition system," Discrete Dynamics in Nature and Society, vol. 2014, Article ID 193143, 7 pages, 2014.

[8] M. H. Protter and H. F. Weinberger, Maximum Principles in Differential Equations, Springer, New York, NY, USA, 1984.

[9] J. Mawhin, H. B. Thompson, and E. Tonkes, "Uniqueness for boundary value problems for second order finite difference equations," Journal of Difference Equations and Applications, vol. 10, no. 8, pp. 749-757, 2004.

[10] P. Stehlík and B. Thompson, "Maximum principles for second order dynamic equations on time scales," Journal of Mathematical Analysis and Applications, vol. 331, no. 2, pp. 913-926, 2007.

[11] P. Stehlík and J. Volek, "Transport equation on semidiscrete domains and Poisson-Bernoulli processes," Journal of Difference Equations and Applications, vol. 19, no. 3, pp. 439-456, 2013.

[12] A. Slavík and P. Stehlík, "Dynamic diffusion-type equations on discrete-space domains," Journal of Mathematical Analysis and Applications, vol. 427, no. 1, pp. 525-545, 2015.

[13] M. Friesl, A. Slavík, and P. Stehlík, "Discrete-space partial dynamic equations on time scales and applications to stochastic processes," Applied Mathematics Letters, vol. 37, pp. 86-90, 2014.

[14] A. Slavík, "Product integration on time scales," Dynamic Systems and Applications, vol. 19, no. 1, pp. 97-112, 2010.

[15] J. Volek, "Maximum and minimum principles for nonlinear transport equations on discrete-space domains," Electronic Journal of Differential Equations, vol. 2014, no. 78, pp. 1-13, 2014.

[16] M. Picone, "Maggiorazione degli integrali delle equazioni totalmente paraboliche alle derivate parziali del secondo ordine," Annali di Matematica Pura ed Applicata, vol. 7, no. 1, pp. 145192, 1929.
[17] E. E. Levi, "Sullequazione del calore," Annali di Matematica Pura ed Applicata, vol. 14, no. 1, pp. 187-264, 1908.

[18] L. Nirenberg, "A strong maximum principle for parabolic equations," Communications on Pure and Applied Mathematics, vol. 6, no. 2, pp. 167-177, 1953.

[19] H. F. Weinberger, "Invariant sets for weakly coupled parabolic and elliptic systems," Rendiconti di Matematica, vol. 8, pp. 295310, 1975.

[20] W. G. Kelley and A. C. Peterson, The Theory of Differential Equations: Classical and Qualitative, Springer, New York, NY, USA, 2010.

[21] E. Hairer, S. P. Nørsett, and G. Wanner, Solving Ordinary Differential Equations I: Nonstiff Problems, Springer, Berlin, Germany, 2007. 


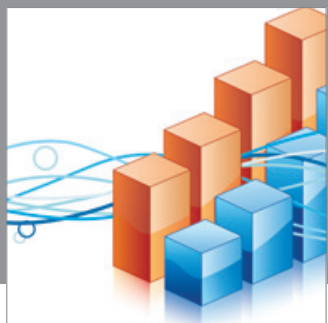

Advances in

Operations Research

mansans

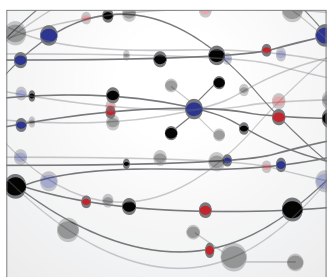

The Scientific World Journal
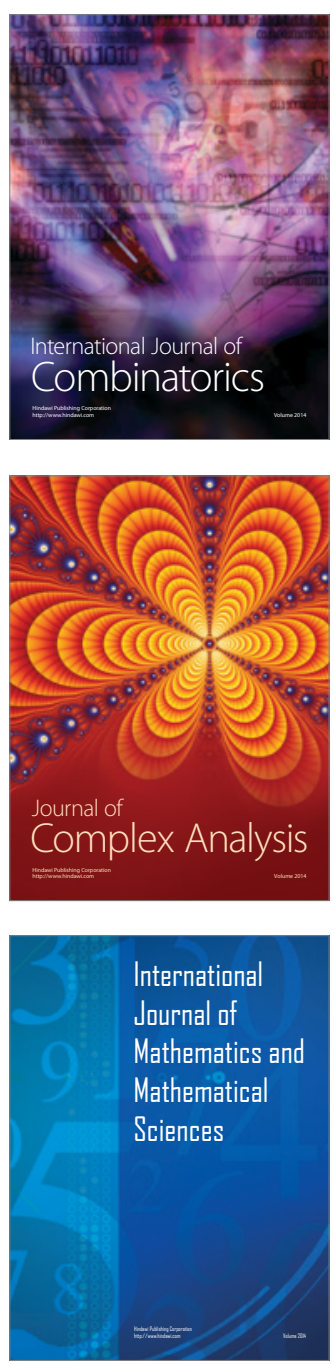
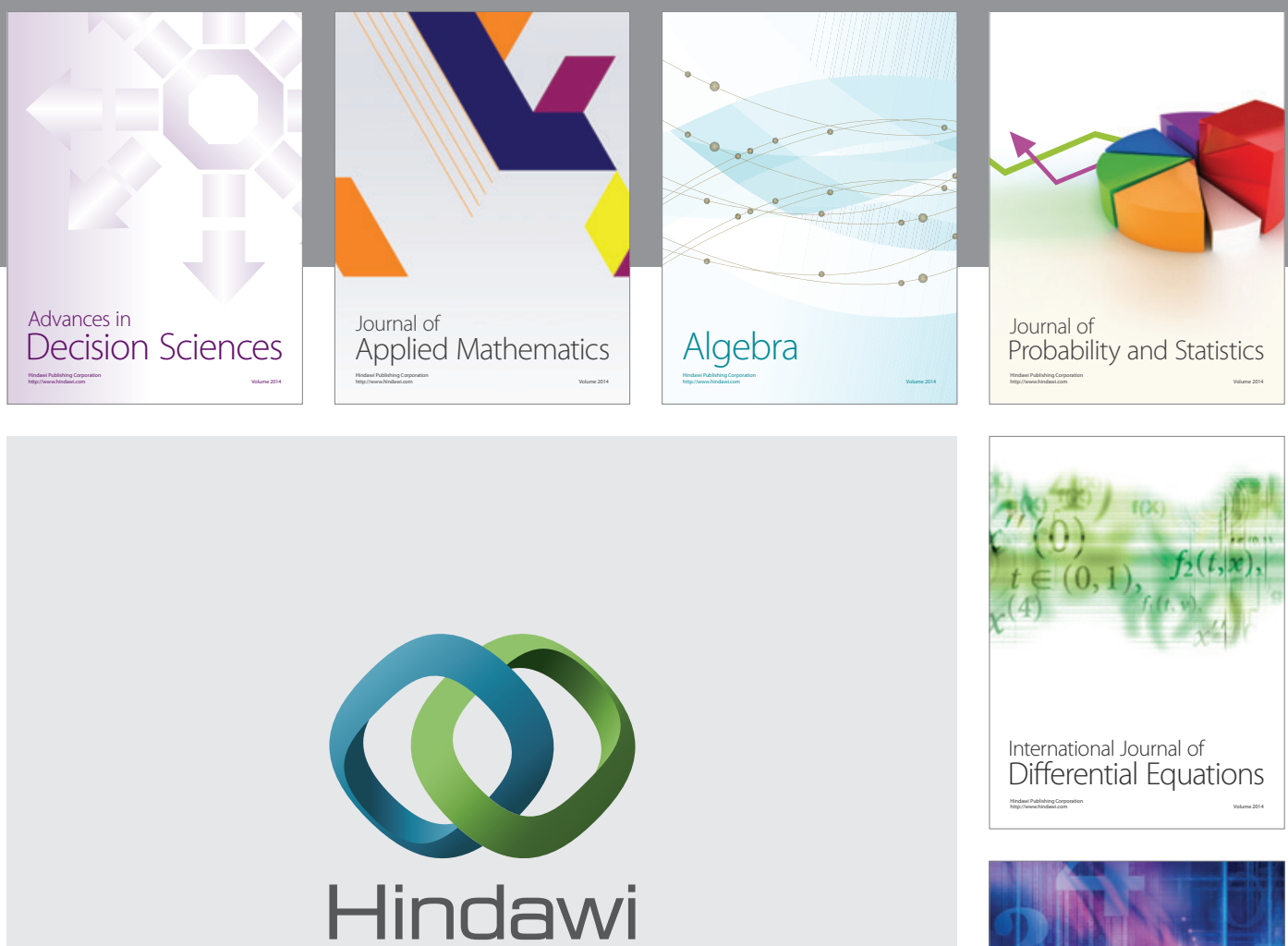

Submit your manuscripts at http://www.hindawi.com
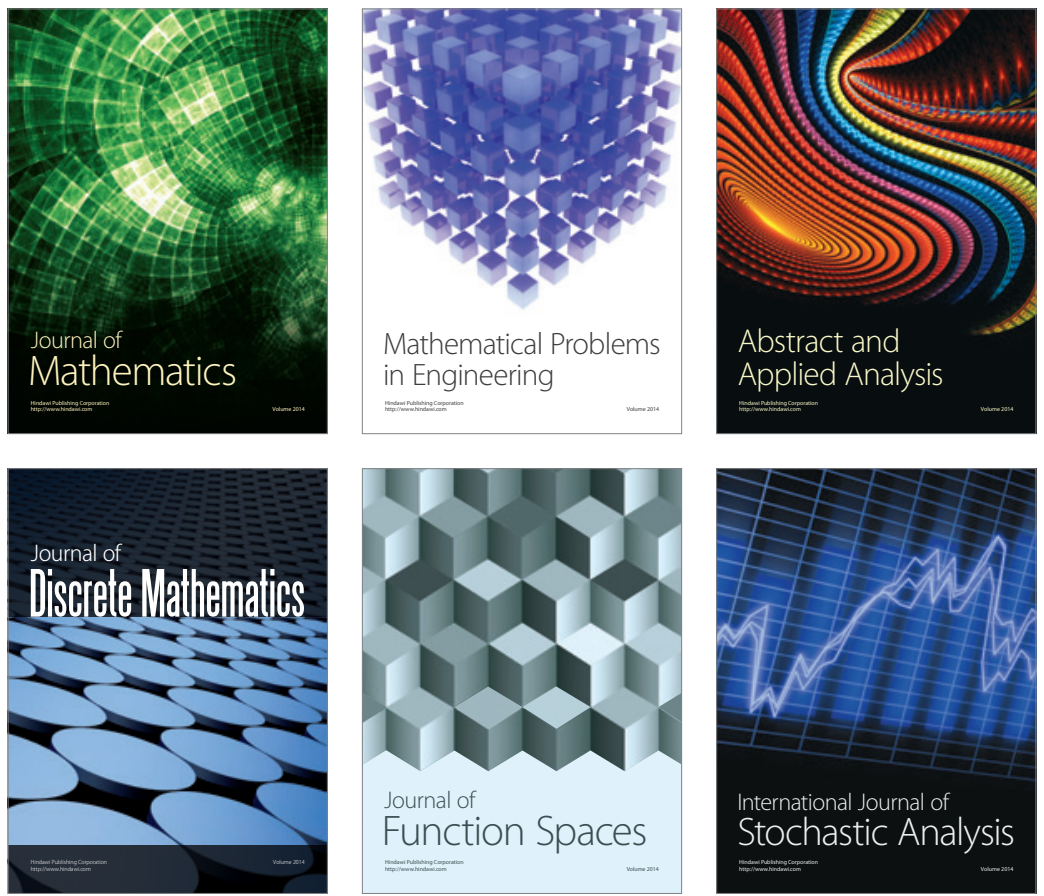

Journal of

Function Spaces

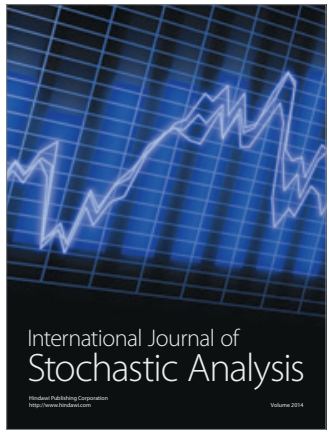

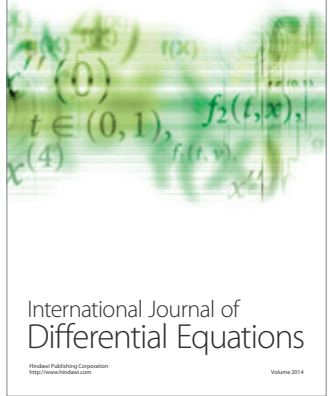
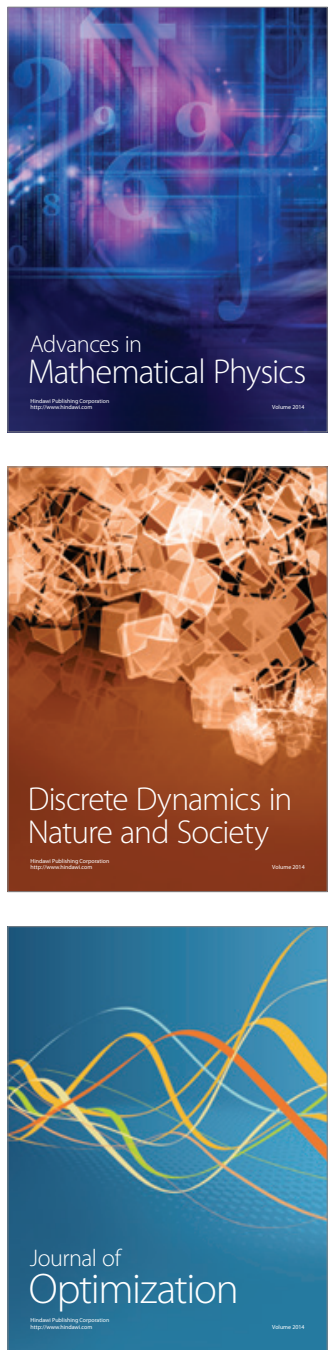